\title{
Laparoscopic distal pancreatectomy for benign and malignant disease: a review of techniques and results
}

\author{
Danilo Coco ${ }^{1}$, Silvana Leanza ${ }^{2}$, Riccardo Schillaci ${ }^{3}$, Giuseppe Angelo Reina ${ }^{4}$ \\ ${ }^{1}$ Department of General Surgery, Ospedali Riuniti Marche Nord, Pesaro, Italy \\ ${ }^{2}$ Department of General Surgery, Carlo Urbani Hospital, Jesi, Ancona, Italy \\ ${ }^{3}$ Department of General Surgery, Biancavilla Hospital, Biancavilla, Italy \\ ${ }^{4}$ Department of General Surgery, Paterno' Biancavilla Hospital, Paternò Biancavilla, Italy
}

Gastroenterology Rev 2022; 17 (2): 103-109

DOI: https://doi.org/10.5114/pg.2021.109625

Key words: pancreatic cancer, laparoscopic distal pancreatectomy, open distal pancreatectomy, radical antegrade modular pancreatosplenectomy, Warshaw technique, Kimura technique. Address for correspondence: Dr Danilo Coco, Department of General Surgery, Ospedali Riuniti Marche Nord, Pesaro, Italy,
phone: +393400546021, e-mail: webcostruction@msn.com

\begin{abstract}
Diagnosis of pancreatic cancer is challenging in the initial phases because its progression is rapid. The pancreatic tail and body roughly accounts for 20-30\% of all cancerous cases. The standard treatment for symptomatic benign, malignant, and premalignant diseases of the pancreatic tail and body is distal pancreatectomy. This technique has been modified over the years to fit certain indications, with the goal of enhancing post-operative results as well as reducing patient trauma. In cases of a premalignant and symptomatic benign condition, the spleen must be preserved either using Kimura's splenic vessel preservation technique or Warshaw's splenic vessel resection technique. A better long-term prognosis is ensured by regional lymph node dissection and radical RO resection. Radical antegrade modular pancreatosplenectomy was proposed to tackle the shortcomings of traditional surgery for pancreatic tail and body cancer. In this review, study techniques and results of laparoscopic distal pancreatectomy for malignant and benign pancreatic ailments were described with the intention of providing knowledge on various suitable techniques reported for pancreatic cancer treatment. Furthermore, this study will serve as a ready reckoner for surgeons and could serve to boost their confidence levels during surgery by avoiding confusion on the selection of suitable for the pancreatic diseases diagnosed.
\end{abstract}

\section{Introduction}

The standard treatment for malignant, premalignant, and symptomatic benign lesions in the pancreatic tail and body is distal pancreatectomy. In the case of malignant illness, a distal pancreatectomy entails pancreatic tissue resection to the left of the portomesenteric vein, which can be extended with lymphadenectomy and splenectomy. This method has been modified over the years to fit certain indications, with the goal of enhancing post-operative results as well as reducing patient trauma. In comparison to open surgery, an approach of laparoscopy to distal pancreatectomy has been related to lesser post-operative morbidity and shorter hospital stay $[1,2]$. However, this method is assumed to be technically challenging compared to traditional surgical procedures.
Efforts should be made to preserve the spleen in cases of premalignant or symptomatic benign illness, either by preserving splenic vessels as specified by Kimura et al. [3] or by splenic vessel resection as defined by Warshaw [4], because splenectomy is incapacitating for subjects due to the need of antibiotics and vaccinations for the prevention of potentially fatal post-splenectomy sepsis. Also, after splenectomy, antibiotic prophylaxis is recommended by many guidelines to prevent overwhelming post-splenectomy sepsis [5].

There has been a quantum leap from laparotomy to laparoscopic to robotic-assisted pancreatectomy with the advancement of technology. For decades, procedures that were consistent with oncology principles for obtaining an accurate prognosis were studied with regard to malignant tumours in the pancreatic tail or body $[6,7]$. 
One of the most difficult surgical measures in $a b$ dominal surgery is pancreatic surgery [8]. Some anatomical drawbacks, like complex proximity to major retroperitoneal and vasculature locations, held back the laparoscopic procedural application in the pancreatic area [9]. With the advancement of skills in surgery and laparoscopy instrumentation, laparoscopic distal pancreatectomy (LDP) has been used widely for the treatment of low-grade malignancies or benign lesions in the pancreatic tail and body [10].

Concomitant splenectomy was regularly carried out at the initial LDP stage for the sake of technological simplicity. Researchers revealed that removing the spleen put subjects at risk of serious complications like overwhelming post-splenectomy infection (OPSI) and post-operative thrombocytosis, which coincided with a greater understanding of the spleen's immunological role over the years [11-13]. In recent clinical practice, surgeons prefer preservation of the spleen whenever feasible. Patients not only gain from minimal invasion and faster recovery after the surgical procedure, but also their long-term quality of life is significantly improved after laparoscopic spleen-preserving distal pancreatectomy (LSPDP) [14].

Spleen-preserving distal pancreatectomy (SPDP) can be performed either with spleen vessel preservation or resection. Warshaw of Massachusetts General Hospital was the first to report SPDP with the splenic vein and artery ligation in 1988 [4]. Doctor Warshaw's surgical treatment was eventually named after him. In 1996, for the very first time, Kimura et al. of Yamagata University reported splenic vessel preservation (SVP) in spleen-preserving distal pancreatectomy [3]. Diverting multiple short branches from the splenic artery and vein spreading to the tail and body of the pancreas is an inevitable technical issue while performing splenic vessel preservation, which necessitates specific care and requires a lot of time. Many laparoscopic surgeons have favoured the Warshaw method in recent years because it is relatively convenient to operate. However, past research has associated the Warshaw method with an increased risk of stomach varices and splenic infarction, as well as theoretical gastrointestinal bleeding risk during long-term follow-up $[15,16]$. During distal pancreatectomy, spleen preservation is gaining appreciation as the concept of precise surgical procedures is advancing. Despite advancements in potential therapeutic approaches, post-operative pancreatic fistula (POPF) continues to be a surgical complication begging for greater intervention.

Short-term results of SVP and Warshaw operations were found to be different when studies were compared [17-19]. It is worth noting that the long-term effects of the Warshaw technique, particularly on the immunological functioning of preserved spleens, have received little attention. With these viewpoints, the present study is conducted to review the available literature reports to outline the techniques and results of LDP (laparoscopic distal pancreatectomy) for malignant and benign pancreatic disorders.

\section{Laparoscopic distal pancreatectomy (LDP)}

Only in the event of incapacitating symptoms should benign neoplasms of the distal pancreas be treated. The resection of premalignant neoplasms is a must for preventing cancer from progressing. Surgery is the only possible curative treatment option for pancreatic cancer. Distal pancreatectomy was a relatively uncommon procedure until lately, owing to the low pancreatic tumour incidence and high unresectable disease proportion at first presentation. The total number of distal pancreatectomies conducted each year has augmented in recent years, which is assumed to be chiefly due to increased usage and higher diagnostic imaging quality and has resulted in subsequent pancreatic cyst detection [20]. The laparoscopic method to distal pancreatectomy is becoming more popular, and it is now regarded by some as a standard method for distal pancreatectomy for premalignant and benign ailment [21]. However, because this treatment is technically challenging, it must only be conducted by surgeons who have adequate experience with both advanced laparoscopic gastrointestinal surgery and open pancreatic surgery [22].

\section{Techniques}

The technique of LDP can vary greatly between different centres and surgeons. Numerous investigations have shown low intra-operative loss of blood, low rates of conversion, and short operational timeframes [23-26]. In numerous aspects, the method described here was similar. Four to 5 trocars are positioned in a semi-circular way centred around an umbilical camera, as shown in Figure 1. The opening of the gastrocolic ligament should be done using an energy device whilst the gastroepiploic vessels are to be preserved. The stomach is lifted with a suture allowing accessibility to the lesser sac as well as good pancreatic exposure. The Figure 1 shows 2 large, non-tied suture bites through the posterior fundus that are then led out next to the epigastric port $[23,24]$. A laparoscopic retractor device can also be used to retract the stomach otherwise. At this stage, intra-operative ultrasound is used to determine the lesion's exact position, its relationship with splenic vessels, as well as the resection level required. The pancreas is then mobilized once the mobilization 
of the inferior pancreatic border is done, making it accessible to the surface of the posterior pancreas. The superior pancreatic border is dissected further to allow placement of nylon tape around the pancreas. The use of this nylon tape is made to demarcate surgical planes and permit greater mobility.

During LDP, the best choice is transection of the pancreas first and then use of ultrasonic/sealing or/ and electrocautery equipment to separate splenic vessels from the pancreatic specimen in a medial to lateral approach. Transection of small vessels from the splenic vein or artery to the pancreas is possible. This should be done with caution because bleeding posterior to the pancreas might be difficult to control and demands blockage of the splenic blood supply. The surgical plain is delineated by slinging the pancreas on both sides of the tumour with nylon tape and splenic vessels with vessel loops, ensuring enhanced peripancreatic tissue surveillance. The use of this procedure permits easy seclusion of the splenic vessels and pancreas from surrounding structures, making pancreatic transection simpler while avoiding damage to the main vessels [25-28]. The use of a laparoscopic $90^{\circ}$ serrated grasper facilitates slinging the splenic vessels with vessel loops. A lateral-to-medial dissection can be tried if the lesion is situated distally in the tail.

\section{Results}

In comparison to open distal pancreatectomy (ODP), laparoscopic distal pancreatectomy with its widespread global use has been shown to be linked with better peri-operative results, in addition to less intra-operative loss of blood and shorter hospital stay. Subjects who underwent LDP had a shorter hospital stay than those who had an open resection. Furthermore, although LDP causes less loss of blood in patients, careful selection is crucial because subjects who require conversion may have increased complication rates as well as pancreatic leakage [1, 26, 29-33]. Emerging research articles also show that laparoscopic distal pancreatectomy leads to a decrease in post-operative morbidity $[1,32,33]$.

In the present literature, there are just 2 randomised controlled trials (RCTs) comparing ODP with LDP so far. De Rooij reported a single-blind, multicentre RCT (laparoscopic vs. open pancreatoduodenectomy for pancreatic or periampullary tumours (LEOPARD)). Subjects suffering from pancreatic tail or body tumours lacking vascular invasion were separated randomly into ODP and minimally invasive distal pancreatectomy (MIDP) groups. During minimally invasive distal pancreatectomy, the outcomes showed less loss of blood as well as a lengthier operating period [34]. Bjornsson reported on another study, which was an unblinded, parallel-group,

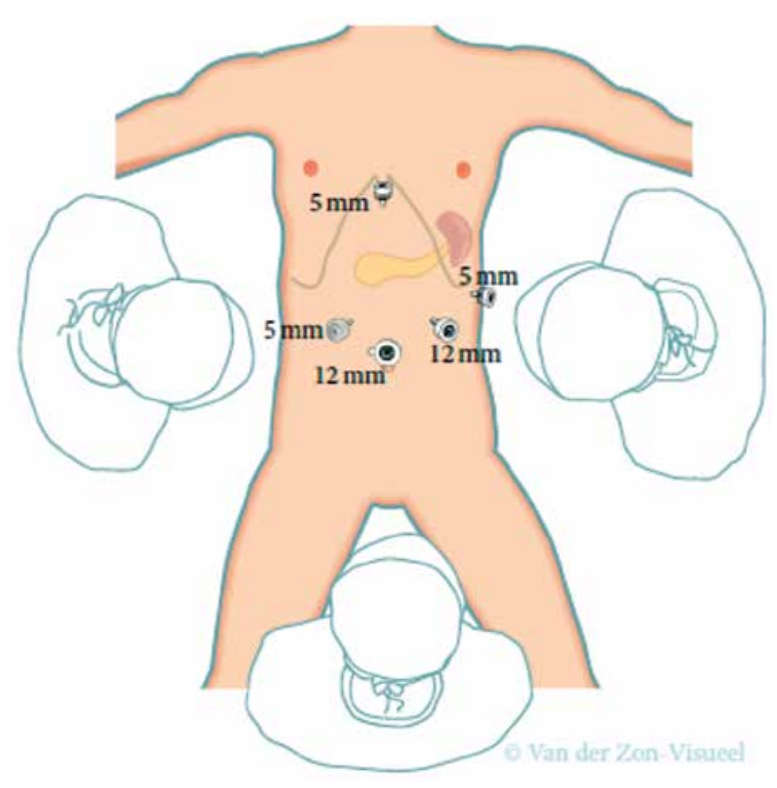

Figure 1. Trocar placement for laparoscopic distal pancreatectomy. The use of a transparent $5 \mathrm{~mm}$ trocar, which is an additional trocar, is suggested during LDP for cancer because it enables easier lymphadenectomy at the celiac trunk and hepatic artery. Source: De Rooij et al. 2015 [27]

single-centre superiority trial. In this research, the laparoscopic distal pancreatectomy group showed comparable benefits with regards to a post-operative hospital stay as well as intra-operative loss of blood [35]. Minimally invasive distal pancreatectomy was proven to reduce the functional recovery time and improve life quality with no increase in hospitalisation cost in comparison to open distal pancreatectomy, as per the outcomes of 2 randomised controlled trials. Both randomised controlled trials, however, failed to show that laparoscopic distal pancreatectomy has a benefit in terms of morbidity. Table I lists the short-term results of ODP and LDP as reported by several researchers in the literature [36-39].

\section{Radical antegrade modular pancreatosplenectomy (RAMPS)}

Diagnosis of pancreatic cancer in the early stages is difficult because its progression is rapid. The pancreatic tail and body account for around $20-30 \%$ of all cases of cancer [40]. Because regional dissection of lymph nodes and radical $\mathrm{RO}$ resection result in improved longterm prognosis, techniques in achieving this aim via standardized surgical approaches have become a hot issue lately. In 2003, Strasberg proposed radical antegrade modular pancreatosplenectomy to overcome the constraints of a conventional surgical method for tail 


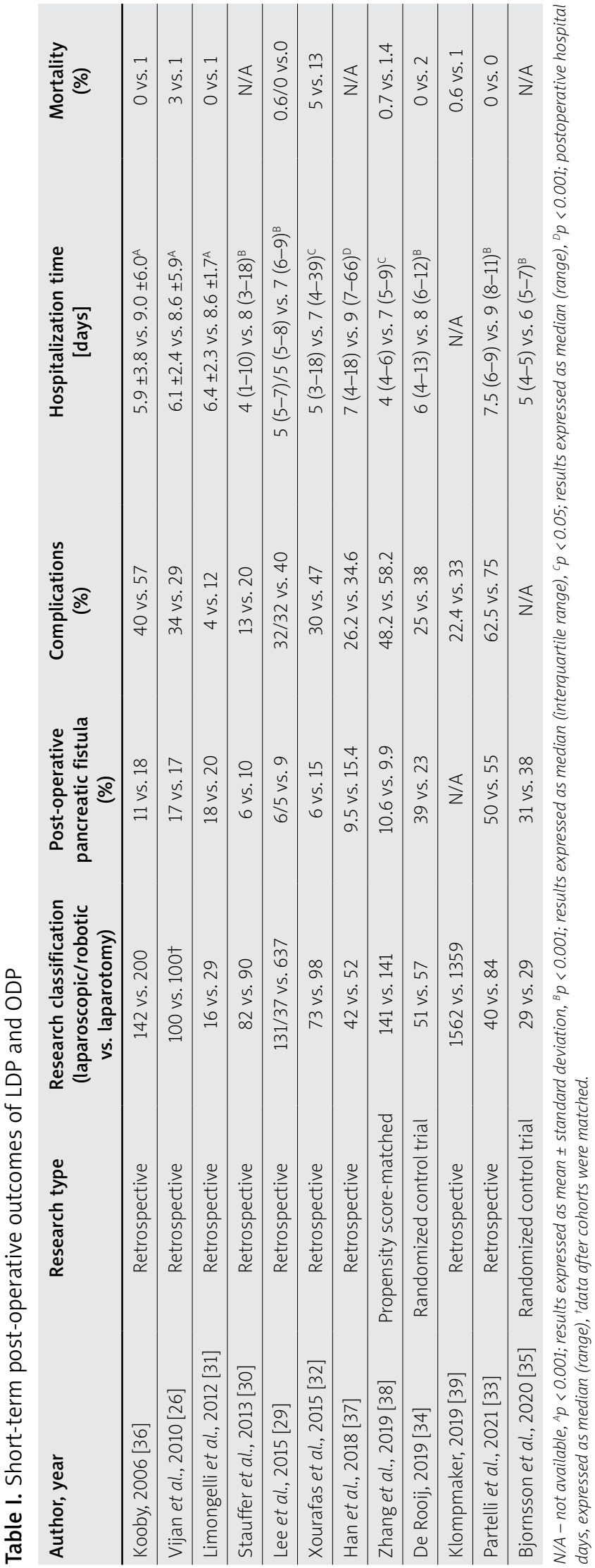

and body cancer of the pancreas. Positive margins were discovered as a result of unsatisfactory retroperitoneal dissection, poor intra-operative bleeding control, as well as insufficient lymphadenectomy [41].

\section{Techniques}

In contrast to a conventional radical surgical method for tumours sited in the pancreatic tail or body, radical antegrade modular pancreatosplenectomy uses a "medial to lateral" technique. The pancreatic neck is transected first, then the splenic vein and artery are ligated at the root, and the left lymph nodes of the celiac trunk and superior mesenteric artery are then dissected. The entire sample, including the pancreatic tail and body, left anterior renal fascia, and spleen, undergoes resection along the left renal vein surface [42].

\section{Results}

The efficacy and safety of RAMPS were indicated in a retrospective study, and the findings revealed that the $\mathrm{RO}$ resection, as well as 5-year survival rates, were satisfactory. The RAMPS outcomes improved greatly in comparison to the traditional surgical method [42]. Other large series have also recommended that radical antegrade modular pancreatosplenectomy can increase the total number of retrieved lymph nodes as well as the negative margin rate; hence, facilitating radical resection and prolonging survival [43]. As a modification of standard retrograde pancreatosplenectomy (SRPS), a comparison of radical antegrade modular pancreatosplenectomy was done with SRPS, and the short-term results indicate a reduced intra-operative loss of blood, improved retrieval of lymph nodes, and the time of operation was shorter with radical antegrade modular pancreatosplenectomy. Additionally, more frequent RO resection than SRPS was also shown. Furthermore, RAMPS also had a better rate of survival than SRPS [44].

Technically, exposing the dissection plane around the deep organs under the left costal margin can be challenging. This raises the possibility of insufficient oncologic dissection risk. To solve this, Watanabe et al. proposed the left kidney mobilisation method. This was proven for being oncologically sound as well as safe in lateral retroperitoneal dissection during radical antegrade modular pancreatosplenectomy for distal pancreatic cancer [45]. With the exception of pancreatic cancer, radical antegrade modular pancreatosplenectomy has produced better oncological results for all pancreatic tumours. Therefore, when there is no availability of pre-operative histological diagnosis, RAMPS could be recommended for all operative tumours that involve the pancreatic tail and body [46]. 


\section{Kimura and Warshaw techniques for spleen-preserving distal pancreatectomy}

In the case of malignant illness of distal pancreas, distal pancreatectomy with subsequent splenectomy is recommended, to guarantee substantial lymph node resection situated along the splenic artery and the splenic hilum [47, 48]. Also, splenectomy is commonly practiced for technical motives, like the involvement of vascular tumours, but mostly because preservation of the spleen can be challenging technically. In the situation of non-malignant illness, however, spleen preservation is recommended, because preservation of the spleen has been linked to reduced peri-operative infections as well as shorter hospital stay [5, 49-51]. As per many systematic evaluations, the introduction of the laparoscopic method in distal pancreatectomy is already linked to increased spleen-preserving distal pancreatectomy (SPDP) rates, with an odds ratio of 3 for laparoscopy in comparison to open surgery [52-54]. Even though the actual cause is unknown, it has been claimed that an improved view during laparoscopic surgery, which allowed better splenic vessel visualisation, led to this finding.

Kimura et al. described an SPDP technique in 1996, which included splenic vein and artery preservation [3]. The researchers came to the conclusion that the method is safe and easy. Contrary to this declaration, this spleen-preservation procedure is recognized to be technically demanding, because the dissection of splenic vessels should be performed circumferentially and should be carefully separated from the pancreas. This method is recommended for proven benign illness. In the situation of premalignant ailment, this technique is recommended when there is no attachment of the lesion to the splenic vessels or spleen; otherwise, a subsequent splenectomy or a SPDP with splenic vessel resection is required.

Warshaw introduced an SPDP with splenic vessel transection in 1988 [4]. The transection of the splenic vein and artery is performed along the side of the portomesenteric vein and at the splenic hilum during this technique, which is now known as the Warshaw method. Staplers or Hem-o-lok clips can be used for vascular control $[24,55]$. It is vital to note that by using 2 Hem-o-lok clips, the vessels can be secured. To prevent the clips from slipping off, a sufficient vascular cuff must be left.

It is still unclear whether the Warshaw or Kimura technique is superior [56-58]. However, a systematic review conducted by Jain et al. reported more issues related to the spleen after the Warshaw method than after the Kimura method, which includes the necessity of post-operative splenectomy ( $2 \%$ vs. $0 \%, p=0.001)$, chronic abdominal pain ( $38 \%$ vs. $0 \%, p=0.048)$, and splenic infarction ( $22 \%$ vs. $2 \%, p=0.001)$ [59]. Thus, the Kimura procedure must be attempted first, with the short gastric arteries being preserved initially. If there is no feasibility for this approach, the Warsaw procedure can be carried out. These recommendations are supported further by recent multicentre retrospective research conducted by Paiella et al. wherein if a Kimura technique does not seem feasible during SPDP, a Warshaw technique was suggested instead of conducting splenectomy [60]. The transection of splenic vessels is done in this circumstance. The left gastroepiploic artery must be preserved because it is assumed to play a significant role in preventing post-operative splenic ischaemia. A vascular endo stapler is also a viable choice for transecting vessels; however, it might be too bulky. Again, clipping and transection of the splenic vessels is made much easier by slinging them with vessel loops. When using the Warshaw procedure for spleen preservation, a bulldog arterial clamp can optionally be used, which reduces splenic blood flow temporarily. Alternatively, the splenic artery can subsequently be dissected medially to the level of the potential pancreatic resection line before pancreatic transection during this technique [25].

During the spleen-preservation method, splenic perfusion must always be examined by the surgeon at the procedural end. A splenectomy must be carried out when splenic ischaemia symptoms, such as extensive ischaemic zones, are evident. Ten per cent of subjects may experience this [56]. Ebihara et al., in their prospective study, found that in the case of doubts about splenic vascularity perfusion during the Warshaw and Kimura laparoscopic techniques, the use of IndoCyanine Green (ICG) can help reduce post-operative complications related to the spleen. The study, with the use of this technique, did not show ischaemia or postoperative splenic abscesses [61].

\section{Conclusions}

Laparoscopic distal pancreatectomy has been indicated to provide improved peri-operative outcome viz. reduced intra-operative loss of blood, a shorter period of stay in the hospital, and decreased post-operative morbidity prognosis over open distal pancreatectomy technique for premalignant and benign pancreatic ailments. Radical antegrade modular pancreatosplenectomy can increase the negative margin rate and the total number of retrieved lymph nodes, thereby facilitating radical resection as well as prolonged survivability. Hence, RAMPS could be recommended for all tumours that are operable involving the pancreatic tail and body 
when there is no availability of a pre-operative histological diagnosis. The SPDP techniques, i.e. Warshaw and Kimura procedures, are recommended for malignant pancreatic diseases and have short-term and longterm results that are comparable. If a Kimura technique does not appear to be possible during SPDP, a Warshaw procedure would be suggested instead of conducting a splenectomy. In the case of perfusion concerns, the use of ICG is recommended.

\section{Conflict of interest}

The authors declare no conflict of interest.

\section{References}

1. Venkat R, Edil BH, Schulick RD, et al. Laparoscopic distal pancreatectomy is associated with significantly less overall morbidity compared to the open technique: a systematic review and meta-analysis. Ann Surg 2012; 255: 1048-59.

2. Nakamura M, Nakashima H. Laparoscopic distal pancreatectomy and pancreatoduodenectomy: is it worthwhile? A meta-analysis of laparoscopic pancreatectomy. J Hepatobiliary Pancreat Sci 2013; 20: 421-8.

3. Kimura W, Inoue T, Futakawa $\mathrm{N}$, et al. Spleen-preserving distal pancreatectomy with conservation of the splenic artery and vein. Surgery 1996; 120: 885-90.

4. Warshaw AL. Conservation of the spleen with distal pancreatectomy. Arch Surg 1988; 123: 550-3.

5. Davies JM, Lewis MP, Wimperis J, et al. Review of guidelines for the prevention and treatment of infection in patients with an absent or dysfunctional spleen: prepared on behalf of the British Committee for Standards in Haematology by a working party of the Haemato Oncology task force. $\mathrm{Br} J$ Haematol 2011; 155: 308-17.

6. Bockhorn M, Uzunoglu FG, Adham M, et al. Borderline resectable pancreatic cancer: a consensus statement by the International Study Group of Pancreatic Surgery (ISGPS). Surgery 2014; 155: 977-88.

7. Sbh F. Clinical Practice Guidelines in Oncology: Bioceramics: for materials science and engineering. 2017: 1-37.

8. Søreide K, Labori KJ. Risk factors and preventive strategies for post-operative pancreatic fistula after pancreatic surgery: a comprehensive review. Scand J Gastroenterol 2016; 51: 1147-54.

9. Poves I, Burdío F, Morató O, et al. Comparison of peri-operative outcomes between laparoscopic and open approach for pancreatoduodenectomy: the PADULAP randomized controlled trial. Ann Surg 2018; 268: 731-9.

10. Mehrabi A, Hafezi M, Arvin J, et al. A systematic review and meta-analysis of laparoscopic versus open distal pancreatectomy for benign and malignant lesions of the pancreas: it's time to randomize. Surgery 2015; 157: 45-55.

11. Schloricke E, Nolde J, Hofmann M, et al. Laparoscopic spleen-preserving distal pancreatectomy. Langenbeck's Arch Surg 2011; 396: 1119-23.

12. Dai MH, Shi N, Xing C, et al. Splenic preservation in laparoscopic distal pancreatectomy. J Br Surg 2017; 104: 452-62.
13. Shi N, Liu SL, Li YT, et al. Splenic preservation versus splenectomy during distal pancreatectomy: a systematic review and meta-analysis. Ann Surg Oncol 2016; 23: 365-74.

14. Kim HS, Park JS, Yoon DS. True learning curve of laparoscopic spleen-preserving distal pancreatectomy with splenic vessel preservation. Surg Endosc 2019; 33: 88-93.

15. Kim H, Song KB, Hwang DW, et al. A single-center experience with the laparoscopic Warshaw technique in 122 consecutive patients. Surg Endosc 2016; 30: 4057-64.

16. Choi SH, Kang CM, Kim JY, et al. Laparoscopic extended (subtotal) distal pancreatectomy with resection of both splenic artery and vein. Surg Endosc 2013; 27: 1412-3.

17. Song J, He Z, Ma S, et al. Clinical comparison of spleen-preserving distal pancreatectomy with or without splenic vessel preservation: a systematic review and meta-analysis. J Laparoendosc Adv Surg Techn 2019; 29: 323-32.

18. Partelli S, Cirocchi R, Randolph J, et al. A systematic review and meta-analysis of spleen-preserving distal pancreatectomy with preservation or ligation of the splenic artery and vein. Surgeon 2016; 14: 109-18.

19. Matsushima H, Kuroki T, Adachi T, et al. Laparoscopic spleen-preserving distal pancreatectomy with and without splenic vessel preservation: the role of the Warshaw procedure. Pancreatology 2014; 14: 530-5.

20. de Jong K, Nio CY, Hermans JJ, et al. High prevalence of pancreatic cysts detected by screening magnetic resonance imaging examinations. Clin Gastroenterol Hepatol 2010; 8: 806-11.

21. Cuschieri A, Jakimowicz JJ, van Spreeuwel J. Laparoscopic distal 70\% pancreatectomy and splenectomy for chronic pancreatitis. Ann Surg 1996; 223: 280.

22. National Institute for Health and Clinical Excellence, Laparoscopic Distal Pancreatectomy, 2007, http://www.nice.org.uk/ guidance/IPG204. Last accessed on June 24, 2021.

23. Robinson S, Saif R, Charnley RM, et al. Surgical adjuncts to laparoscopic distal pancreatectomy. Minim Invasive Ther Allied Technol 2011; 20: 369-73.

24. Hilal MA, Jain G, Kasasbeh F, et al. Laparoscopic distal pancreatectomy: critical analysis of preliminary experience from a tertiary referral centre. Surg Endosc 2009; 23: 2743-7.

25. Hilal MA, Takhar AS. Laparoscopic left pancreatectomy: current concepts. Pancreatology 2013; 13: 443-8.

26. Vijan SS, Ahmed KA, Harmsen WS, et al. Laparoscopic vs. open distal pancreatectomy: a single-institution comparative study. Arch Surg 2010; 145: 616-21.

27. De Rooij T, Sitarz R, Busch OR, et al. Technical aspects of laparoscopic distal pancreatectomy for benign and malignant disease: review of the literature. Gastroenterol Res Pract 2015; 2015: 472906.

28. Velanovich V. The lasso technique for laparoscopic distal pancreatectomy. Surg Endosc Other Interv Techn 2006; 20: 1766-71.

29. Lee SY, Allen PJ, Sadot E, et al. Distal pancreatectomy: a single institution's experience in open, laparoscopic, and robotic approaches. J Am Coll Surg 2015; 220: 18-27.

30. Stauffer JA, Rosales-Velderrain A, Goldberg RF, et al. Comparison of open with laparoscopic distal pancreatectomy: a single institution's transition over a 7-year period. Hpb 2013; 15: 149-55.

31. Limongelli P, Belli A, Russo G, et al. Laparoscopic and open surgical treatment of left-sided pancreatic lesions: clinical 
outcomes and cost-effectiveness analysis. Surg Endosc 2012 26: $1830-6$.

32. Xourafas D, Tavakkoli A, Clancy TE, Ashley SW. Distal pancreatic resection for neuroendocrine tumors: is laparoscopic really better than open? J Gastrointest Surg 2015; 19: 831-40.

33. Partelli S, Andreasi V, Rancoita PM, et al. Outcomes after distal pancreatectomy for neuroendocrine neoplasms: a retrospective comparison between minimally invasive and open approach using propensity score weighting. Surg Endosc 2021; 35: 165-73.

34. de Rooij T, van Hilst J, van Santvoort H, et al. Minimally invasive versus open distal pancreatectomy (LEOPARD): a multicenter patient-blinded randomized controlled trial. Ann Surg 2019; 269: 2-9.

35. Björnsson B, Larsson AL, Hjalmarsson C, et al. Comparison of the duration of hospital stay after laparoscopic or open distal pancreatectomy: randomized controlled trial. J Br Surg 2020, 107: $1281-8$

36. Kooby DA. Laparoscopic surgery for cancer: historical, theoretical, and technical considerations. Oncology 2006; 20: 917-27.

37. Han SH, Han IW, Heo JS, et al. Laparoscopic versus open distal pancreatectomy for nonfunctioning pancreatic neuroendocrine tumors: a large single-center study. Surg Endosc 2018 32: 443-9.

38. Zhang XF, Lopez-Aguiar AG, Poultsides G, et al. Minimally invasive versus open distal pancreatectomy for pancreatic neuroendocrine tumors: an analysis from the US neuroendocrine tumor study group. J Surg Oncol 2019; 120: 231-40.

39. Klompmaker S, de Rooij T, Koerkamp BG, et al. International validation of reduced major morbidity after minimally invasive distal pancreatectomy compared with open pancreatectomy. Ann Surg 2019 doi: 10.1097/SLA.0000000000003659.

40. Bockhorn M, Uzunoglu FG, Adham M, et al. Borderline resectable pancreatic cancer: a consensus statement by the International Study Group of Pancreatic Surgery (ISGPS). Surgery 2014; 155: 977-88.

41. Strasberg SM, Drebin JA, Linehan D. Radical antegrade modular pancreatosplenectomy. Surgery 2003; 133: 521-7.

42. Mitchem JB, Hamilton N, Gao F, et al. Long-term results of resection of adenocarcinoma of the body and tail of the pancreas using radical antegrade modular pancreatosplenectomy procedure. J Am Coll Surgeons 2012; 214: 46-52.

43. Grossman JG, Fields RC, Hawkins WG, Strasberg SM. Single institution results of radical antegrade modular pancreatosplenectomy for adenocarcinoma of the body and tail of pancreas in 78 patients. J Hepatobiliary Pancreat Sci 2016; 23: 432-41.

44. Abe T, Ohuchida K, Miyasaka Y, et al. Comparison of surgical outcomes between radical antegrade modular pancreatosplenectomy (RAMPS) and standard retrograde pancreatosplenectomy (SPRS) for left-sided pancreatic cancer. World J Surg 2016; 40: 2267-75.

45. Watanabe G, Ito H, Sato T, et al. Left kidney mobilization technique during radical antegrade modular pancreatosplenectomy (RAMPS). Langenbeck's Arch Surg 2019; 404: 247-52.

46. Sivasanker $M$, Desouza A, Bhandare $M$, et al. Radical antegrade modular pancreatosplenectomy for all pancreatic body and tail tumors: rationale and results. Langenbeck's Arch Surg 2019; 404: 183-90.
47. Tol Johanna AM, Gouma Dirk J, Bassi C, et al. Definition of a standard lymphadenectomy in surgery for pancreatic ductal adenocarcinoma: a consensus statement by the International Study Group on Pancreatic Surgery (ISGPS). Surgery 2014; 156 : 591-600.

48. Strasberg SM, Linehan DC, Hawkins WG. Radical antegrade modular pancreatosplenectomy procedure for adenocarcinoma of the body and tail of the pancreas: ability to obtain negative tangential margins. J Am Coll Surgeons 2007; 204: 244-9.

49. Fernández-Cruz L, Orduna D, Cesar-Borges G, López-Boado MA. Distal pancreatectomy: en-bloc splenectomy vs spleen-preserving pancreatectomy. Hpb 2005; 7: 93-8.

50. Shoup M, Brennan MF, McWhite K, et al. The value of splenic preservation with distal pancreatectomy. Arch Surg 2002; 137 : 164-8.

51. Govil S, Imrie CW. Value of splenic preservation during distal pancreatectomy for chronic pancreatitis. J Br Surg 1999; 86: 895-8.

52. Jin T, Altaf K, Xiong JJ, et al. A systematic review and meta-analysis of studies comparing laparoscopic and open distal pancreatectomy. Hpb 2012; 14: 711-24.

53. Jusoh AC, Ammori BJ. Laparoscopic versus open distal pancreatectomy: a systematic review of comparative studies. Surg Endosc 2012; 26: 904-13.

54. Nigri GR, Rosman AS, Petrucciani N, et al. Metaanalysis of trials comparing minimally invasive and open distal pancreatectomies. Surg Endosc 2011; 25: 1642-51.

55. Rehman S, John SK, Lochan R, et al. Oncological feasibility of laparoscopic distal pancreatectomy for adenocarcinoma: a single-institution comparative study. World J Surg 2014; 38: 476-83.

56. Ferrone CR, Konstantinidis IT, Sahani DV, et al. Twenty-three years of the Warshaw operation for distal pancreatectomy with preservation of the spleen. Ann Surg 2011; 253: 1136-9.

57. Butturini G, Inama M, Malleo G, et al. Perioperative and longterm results of laparoscopic spleen-preserving distal pancreatectomy with or without splenic vessels conservation: a retrospective analysis. J Surg Oncol 2012; 105: 387-92.

58. Zhou ZQ, Kim SC, Song KB, et al. Laparoscopic spleen-preserving distal pancreatectomy: comparative study of spleen preservation with splenic vessel resection and splenic vessel preservation. World J Surg 2014; 38: 2973-9.

59. Jain G, Chakravartty S, Patel AG. Spleen-preserving distal pancreatectomy with and without splenic vessel ligation: a systematic review. Hpb 2013; 15: 403-10.

60. Paiella S, De Pastena M, Korrel M, et al. Long term outcome after minimally invasive and open Warshaw and Kimura techniques for spleen-preserving distal pancreatectomy: international multicenter retrospective study. Eur J Surg Oncol 2019; 45: 1668-73.

61. Ebihara Y, Noji T, Tanaka K, et al. Laparoscopic real-time vessel navigation using indocyanine green fluorescence during the laparoscopic-Warshaw technique: first clinical experience. J Minim Access Surg 2021; 17: 226-9.

Received: 26.07.2021

Accepted: 14.08.2021 